\title{
Fuzzy Logic Application to a Two-wheel Mobile Robot for Balancing Control Performance
}

\author{
Hyun Wook Kim and Seul Jung \\ Intelligent Systems and Emotional Engineering (ISEE) Lab. \\ Department of Mechatronics Engineering \\ Chungnam National University, Daejeon, Korea
}

\begin{abstract}
This article presents experimental studies of fuzzy logic application to control a two-wheel mobile robot(TWMR) system. The TWMR system is composed of two systems, an inverted pendulum system and a mobile robot system. Although linear controllers can stabilize the TWMR, fuzzy controllers are expected to have robustness to uncertainties so that the resulting performances are expected to be better. Nominal fuzzy rules are used to control balance and position of TWMR. Fuzzy logic is embedded on a DSP chip to control the TWMR. Balancing performances of the PID controller and the fuzzy controller under disturbances are compared through extensive experimental studies.
\end{abstract}

Keywords :Balancing control, Two-wheel mobile robot, fuzzy control, PID control.

\section{Introduction}

A balancing control task is one of important mechanisms in dynamical systems. Our human body systems are required to maintain balance when standing, walking, and running. In dynamical systems, robust balancing performances are required for stable walking of humanoid robots, balancing the pendulum of inverted pendulum systems and mobile inverted pendulum systems, and hovering of flying systems.

The inverted pendulum system is widely utilized as a model of control education since it is a single-input multiple-output(SIMO) system and has a nonlinear characteristic The goal is to maintain the balance of the pendulum while moving toward the desired position. Controlling both the balancing angle of the pendulum and the position of the cart is not easy due to aforementioned characteristics.

The balancing concept of inverted pendulum systems can be extended to many control applications. A walking motion of humanoid robots can be modeled as several linkages with an inverted pendulum system that considers the movement of the center of gravity of the system [1,2]. The center of gravity compensation method is often used for balancing of walking motions of humanoid robots.

A two-wheel mobile robot(TWMR) is an extended system of an inverted pendulum. The TWMR has challenging structural characteristics of combing two systems, a mobile robot system and an inverted pendulum system. The eventual goal of the TWMR is to maintain the balance of the angle when it navigates on the $\mathrm{x}-\mathrm{y}$ plane toward the destination.

In the control aspect, TWMR is quite a challenging system to

Manuscript received Jan. 3, 2012; revised Jun. 7, 2012; accepted Jun. 15, 2012

*Corresponding Author: Seul Jung(jungs@cnu.ac.kr)

(c) The Korean Institute of Intelligent Systems. All rights reserved. satisfy both requirements of balancing and navigation. Many attempts for controlling TWMR systems have been made in the literature including Segway[3]. Model-based control methods have been presented [4-11]. Although controlling both a balancing angle and a position of TWMR by linear controllers is possible, performances of PID control methods under disturbances are limited for certain conditions such as a symmetrical design.

To remedy the unstable balancing behavior, a neural network has been introduced. Intelligent control techniques such as neural network have been applied to the TWMR as an auxiliary controller to help main linear controllers by improving the robustness against disturbances $[12,13]$.

In other aspects, fuzzy logic is also a good candidate of intelligent tools that can provide its superiority over conventional methods in various areas. Abilities of fuzzy controllers have been proved in many control applications, especially in nonlinear complex system control applications [14]. This leads to the production of fuzzy controlled commercial appliances.

Fuzzy logic is known to have a strong advantage of representing human expression into numerical expression for controlling dynamical systems. Conversely, fuzzy logic requires a time-consuming process to find optimal rules, which is considered as a disadvantage. However, fuzzy controllers perform better than linear controllers since they function as a non linear controller with infinite gains.

Therefore, in this paper, a nominal fuzzy control method is applied to TWMR to test its performance. The fuzzy controller is expected to show better performances by improving the balancing performance and increasing the robustness under disturbance. Experimental studies of balancing control of the TWMR are performed to demonstrate the robust performance. Experimental results are compared with those of linear controllers. 


\section{Modeling of Two-wheel Mobile Robot System}

The kinematic model of TWMR system is shown in Fig.1. Control variables are a balancing angle $\theta$, position $p$, and a heading angle $\phi$. Regulating two wheel velocities, $\dot{\theta}_{r}$ and $\dot{\theta}_{l}$ controls angles and position. Three separate controllers are required for controlling a balancing angle, a heading angle, and a position. $L$ is the distance between two wheels and $r$ is the radius of the wheel.

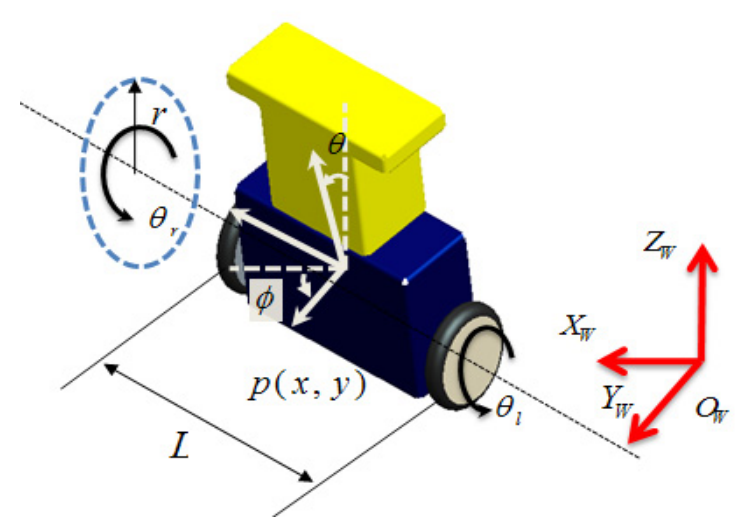

Fig.1. Modelling of two-wheel mobile robot system

The relationship between the Cartesian velocity and the joint velocity yields

$$
\left[\begin{array}{c}
v_{m} \\
\omega_{m}
\end{array}\right]=\left[\begin{array}{cc}
\frac{r}{2} & \frac{r}{2} \\
\frac{r}{L} & -\frac{r}{L}
\end{array}\right]\left[\begin{array}{l}
\dot{\theta}_{R} \\
\dot{\theta}_{L}
\end{array}\right]
$$

where $\dot{\theta}_{R}, \dot{\theta}_{L}$ are angular velocities of the right and left wheel, respectively, and $v_{m}$ is the linear velocity and $\omega_{m}$ is the angular velocity of the TWMR system.

Then we have the kinematics equation of the two-wheel mobile robot system as follows.

$$
\left[\begin{array}{c}
\dot{x} \\
\dot{y} \\
\dot{\phi}
\end{array}\right]=\left[\begin{array}{cc}
\frac{r}{2} \cos \phi & \frac{r}{2} \cos \phi \\
\frac{r}{2} \sin \phi & \frac{r}{2} \sin \phi \\
\frac{r}{L} & -\frac{r}{L}
\end{array}\right]\left[\begin{array}{l}
\dot{\theta}_{R} \\
\dot{\theta}_{L}
\end{array}\right]
$$

where $\dot{x}=v_{m} \cos \phi$ and $\dot{y}=v_{m} \sin \phi$.

Thus, in the framework of the kinematics based control, we can control a balancing angle, a position, and a heading angle of the TWMR by controlling angular velocities of two wheels which are directly related with torques.

\section{Linear Control Scheme}

The PID control method is mostly used in the industrial application because it is simple and easy to implement when the system dynamic model is not available. Torque of the left and the right wheel can be adjusted by PID gains to satisfy the performances.

Here each wheel torque is summed together as shown in Fig. 2. Since the integral action has ill effects on the case of balancing control of TWMR due to the accumulated errors, the PD control method is used for the balancing angle control and the PID control method is used for the position and heading angle control. The right and left torques are computed as below.

Since we assume that TWMR is symmetrical, the desired balancing angle is set to zero. This structural symmetry of the system allows us to use linear controllers to control the system.

$$
\begin{aligned}
& \tau_{R}=K_{p \theta}\left(\theta_{d}-\theta\right)+K_{d \theta}\left(\dot{\theta}_{d}-\dot{\theta}\right) \\
& +K_{p \phi}\left(\phi_{m d}-\phi_{m}\right)+K_{d \phi}\left(\omega_{m d}-\omega_{m}\right)+K_{i \phi} \int_{0}^{t}\left(\phi_{m d}-\phi_{m}\right) d t \\
& +K_{p p}\left(p_{m d}-p_{m}\right)+K_{d p}\left(v_{m d}-v_{m}\right)+K_{i p} \int_{0}^{t}\left(p_{m d}-p_{m}\right) d t \\
& \tau_{L}=K_{p \theta}\left(\theta_{d}-\theta\right)+K_{d \theta}\left(\dot{\theta}_{d}-\dot{\theta}\right) \\
& -K_{p \phi}\left(\phi_{m d}-\phi_{m}\right)-K_{d \phi}\left(\omega_{m d}-\omega_{m}\right)-K_{i \phi} \int_{0}^{t}\left(\phi_{m d}-\phi_{m}\right) d t \\
& +K_{p p}\left(p_{m d}-p_{m}\right)+K_{d p}\left(v_{m d}-v_{m}\right)+K_{i p} \int_{0}^{t}\left(p_{m d}-p_{m}\right) d t
\end{aligned}
$$

where $\tau_{R}$ and $\tau_{L}$ are torque of the right wheel and the left wheel, respectively and $K_{i j}$ is the controller gain.

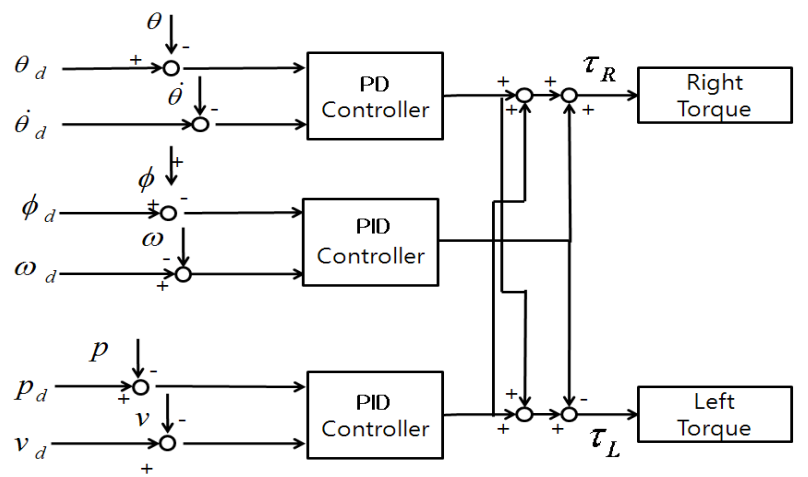

Fig. 2 Linear control block diagram

\section{Fuzzy Control}

A fuzzy controller is a nonlinear controller that can have infinite gains. In the control framework, a fuzzy controller replaces a linear controller to control the TWMR as shown in Fig. 3. The inside procedure of the fuzzy controller includes several processes such as fuzzification, fuzzy inference, and defuzzification. 


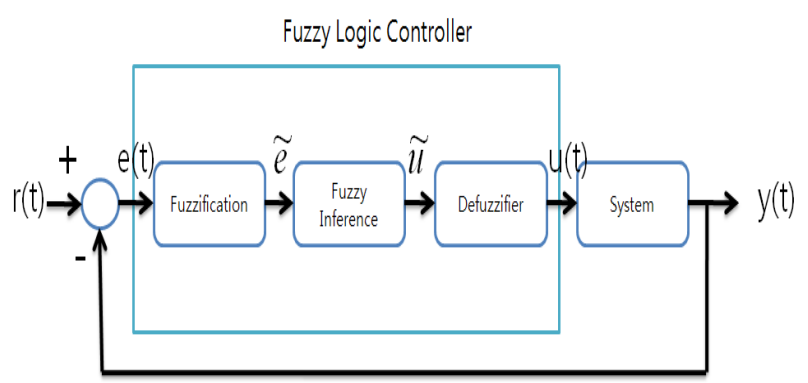

Fig. 3 Fuzzy control block diagram

Inputs to the fuzzy controller are an error $e(t)$ and an error rate $\Delta e(t)$. These numerical data are normalized and converted into fuzzy sets, $\widetilde{e}$ and $\Delta \widetilde{e}$ as shown in Fig. 4 (a). Instead of designing optimal fuzzy rules, nominal fuzzy rules given in Fig. 4(b) are used. Defining relations among fuzzy sets $\widetilde{e}$ and $\Delta \widetilde{e}$ is called fuzzy rules for the control input $\tilde{u}$.

Fig. 4(b) lists fuzzy rules showing the relationship between the fuzzy inputs $\widetilde{e}$ and $\Delta \widetilde{e}$, and the fuzzy output $\widetilde{u}$. Fuzzy rules for the balancing angle, the heading angle, and the position are described as

$$
\begin{aligned}
& R_{\theta} \text { : If } e_{\theta} \text { is } A_{i}, \Delta e_{\theta} \text { is } B_{i} \text {, then } u_{\theta} \text { is } C_{i} \\
& R_{p}: \text { If } e_{p} \text { is } A_{j}, \Delta e_{p} \text { is } B_{j} \text {, then } u_{p} \text { is } C_{j} \\
& R_{\phi}: \text { If } e_{\phi} \text { is } A_{k}, \Delta e_{\phi} \text { is } B_{k} \text {, then } u_{\phi} \text { is } C_{k}
\end{aligned}
$$

One fuzzy rule can be processed as shown in (4).

$$
\begin{aligned}
& \mu_{C_{1}}(u)=\mu_{A_{1}}\left(e_{i}\right) \wedge \mu_{B_{1}}\left(\Delta e_{i}\right) \\
& =\min \left\{\mu_{A_{1}}\left(e_{i}\right) \wedge \mu_{B_{1}}\left(\Delta e_{i}\right)\right\}
\end{aligned}
$$

where $\mu_{A_{1}}$ is the membership value of fuzzy set $A_{1}$.

Several fuzzy sets from corresponding fuzzy rules are obtained. Fuzzy inference process determines a resultant fuzzy set $\tilde{u}$ from several fuzzy sets. Finally, the fuzzy set $\tilde{u}$ is converted into a numerical value for controlling the system. This process is called defuzzification. The center of gravity method as one of defuzzification methods is given in (5).

$$
u=\frac{\sum_{i=1}^{L} \mu_{u}\left(u_{i}\right) \cdot u_{i}}{\sum_{i=1}^{L} \mu_{u}\left(u_{i}\right)}
$$

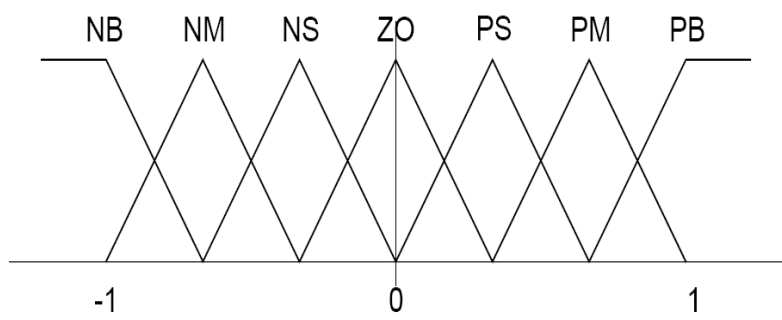

(a) Membership function

\begin{tabular}{|c|l|l|l|l|l|l|l|l|}
\hline \multicolumn{2}{|c|}{} & \multicolumn{7}{|c|}{$\Delta \widetilde{e}$} \\
\cline { 3 - 9 } & NB & $\begin{array}{l}\text { N } \\
\text { M }\end{array}$ & NS & ZO & PS & PM & PB \\
\hline \multirow{5}{*}{$\widetilde{e}$} & NB & PB & PB & PM & PM & PM & PS & ZO \\
\cline { 2 - 9 } & NM & PB & PM & PM & PM & PS & ZO & NS \\
\cline { 2 - 9 } & NS & PM & PM & PM & PS & ZO & NS & NM \\
\cline { 2 - 9 } & ZO & PM & PM & PS & ZO & NS & NM & NM \\
\cline { 2 - 9 } & PS & PM & PS & ZO & NS & NM & NM & NM \\
\cline { 2 - 9 } & PM & PS & ZO & NS & NM & NM & NM & NB \\
\cline { 2 - 9 } & PB & ZO & NS & NM & NM & NM & NB & NB \\
\hline
\end{tabular}

ZO : Zero

PS(NS) : Positive(Negative) Small $\mathrm{PM}(\mathrm{NM})$ : Positive(Negative) Medium $\mathrm{PB}(\mathrm{NB})$ : Positive(Negative) Big

(b) Fuzzy rules

Fig. 4 Membership Function and Fuzzy rule

A detailed fuzzy control block diagram for the TWMR is shown in Fig. 5. Since the TWMR is a combined system of an inverted pendulum system and a mobile robot system, control variables are a position and a heading angle from a mobile robot system and a balancing angle from an inverted pendulum system. The balancing angle, the position, and the heading angle of TWMR are controlled separately by three fuzzy controllers by replacing linear controllers in Fig. 2.

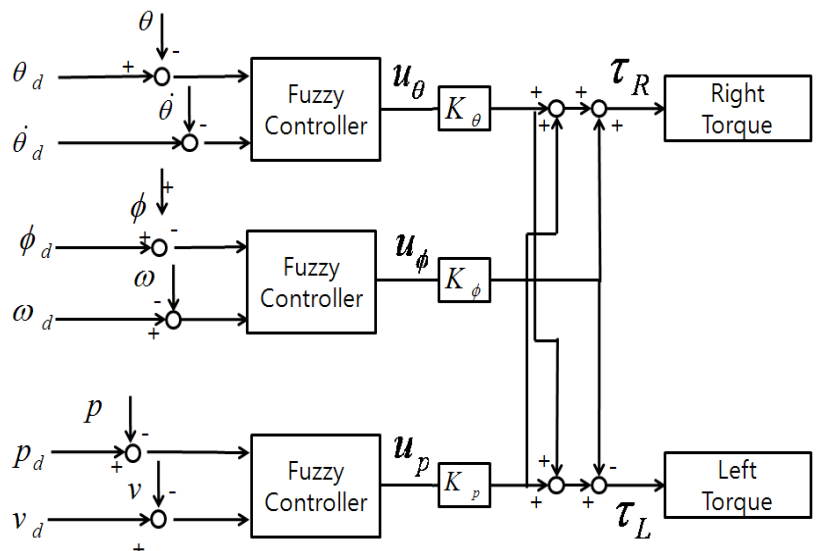

Fig. 5 Fuzzy Control Block Diagram

Since each fuzzy controller output is normalized, it should be denormalized by multiplying a constant gain for actuating torques. Right and left torques of the TWMR are computed as

$$
\begin{aligned}
& \tau_{r}=K_{\theta} u_{\theta}+K_{p} u_{p}+K_{\phi} u_{\phi} \\
& \tau_{l}=K_{\theta} u_{\theta}+K_{p} u_{p}-K_{\phi} u_{\phi}
\end{aligned}
$$

where $u_{\theta}, u_{p}, u_{\phi}$ are fuzzy controller outputs and $K_{\theta}, K_{p}, K_{\phi}$ are constant gains for the balancing angle, the position, and the heading angle, respectively

Input variables $\theta, p, \phi$ and their derivatives have the ranges listed in Table 1. Those ranges are normalized to fit the 
membership functions in Fig. 4 (a). Denormalizing gains used for experimental studies are listed in Table 2.

Table 1. Input variables and their ranges

\begin{tabular}{|c|c|c|c|c|c|c|}
\hline Variable & $\begin{array}{c}\theta \\
(\mathrm{deg})\end{array}$ & $\begin{array}{c}\dot{\theta} \\
(\mathrm{deg} / \mathrm{s})\end{array}$ & $\begin{array}{c}p \\
(\mathrm{~cm})\end{array}$ & $\begin{array}{c}\dot{p} \\
(\mathrm{~cm} / \mathrm{s})\end{array}$ & $\begin{array}{c}\phi \\
(\mathrm{deg})\end{array}$ & $\begin{array}{c}\dot{\phi} \\
(\mathrm{deg} / \mathrm{s})\end{array}$ \\
\hline Range & $-3 \sim 3$ & $-4 \sim 4$ & $-20 \sim 20$ & $-15 \sim 15$ & $-10 \sim 10$ & $-10 \sim 10$ \\
\hline
\end{tabular}

Table 2. Output gains

\begin{tabular}{|c|c|c|c|}
\hline & $K_{\theta}$ & $K_{p}$ & $K_{\phi}$ \\
\hline Gain & 300 & 300 & 50 \\
\hline
\end{tabular}

\section{Real System Integration}

The real robot system is shown in Fig. 6. The whole body is designed to be as symmetrical as possible so that the location of the center of gravity can be placed on the center of the wheel axis. All of components are laid out on the center to make a symmetrical structure.

The main control hardware is the DSP2812 chip that generates PWM signals, collects encoder data, processes sensors, and communicates with a PC. The balancing angle is detected by fusing a gyro and a tilt sensor. Position and heading angles are detected by two encoders. Control sampling time is $100 \mathrm{~Hz}$. The overall hardware structure is described in Fig. 7.

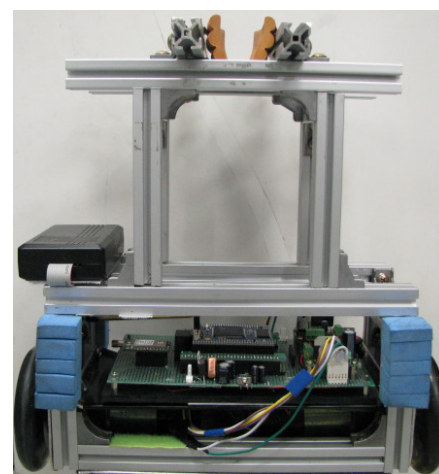

(a) Front view

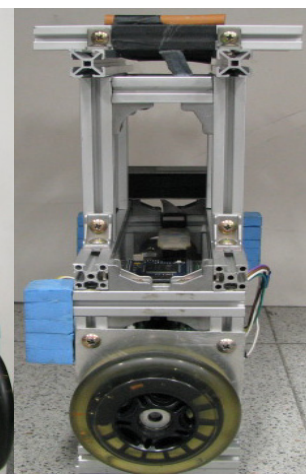

(b) Side view
Fig. 6 Two-wheel mobile robot system

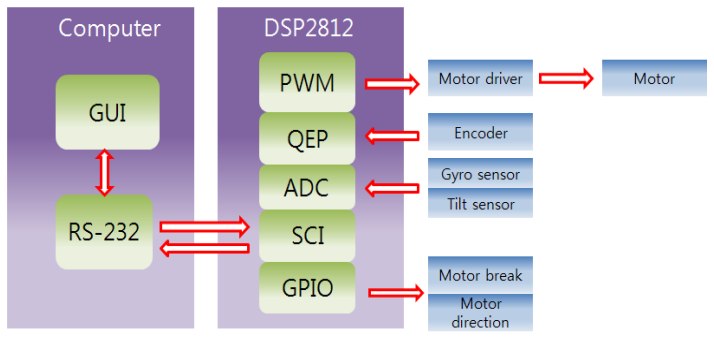

Fig. 7 Overall hardware structure

\section{Experimental Studies}

\subsection{Balancing Control Experiment}

The TWMR is tested to maintain balance using the PID controller and the fuzzy controller. Table 3 shows the PID controller gains used in experimental studies for comparison. PID gains are found experimentally to have the better performance.

\begin{tabular}{|c|c|c|}
\multicolumn{2}{c}{ Table 3 PID gains } \\
\hline $\begin{array}{c}\text { Balancing angle } \\
\text { control }\end{array}$ & $\begin{array}{c}\text { Position } \\
\text { control }\end{array}$ & Heading angle control \\
\hline$K_{p \theta}=110$ & $K_{p p}=-20$ & $K_{p \phi}=16$ \\
$K_{d \theta}=15$ & $K_{d p}=-22$ & $K_{d \phi}=0.2$ \\
& $K_{i p}=-1$ & $K_{i \phi}=0.1$ \\
\hline
\end{tabular}

The first experiment is required to regulate balancing of the TWMR using the linear control and the fuzzy control method. The resulting balancing image cut pictures by the linear controller are shown in Fig. 8. Fig. 8 shows two images of the balancing control demonstration that is captured with a time interval to show that TWMR keeps moving back and forth. Red lines indicate that lines do not align each other. Although the robot keeps oscillating back and forth, TWMR maintains stable balancing. The corresponding plots are shown in Fig. 9. Fig. 9 (b) indicates that TWMR oscillates within the positional error of $\pm 0.03 m$.

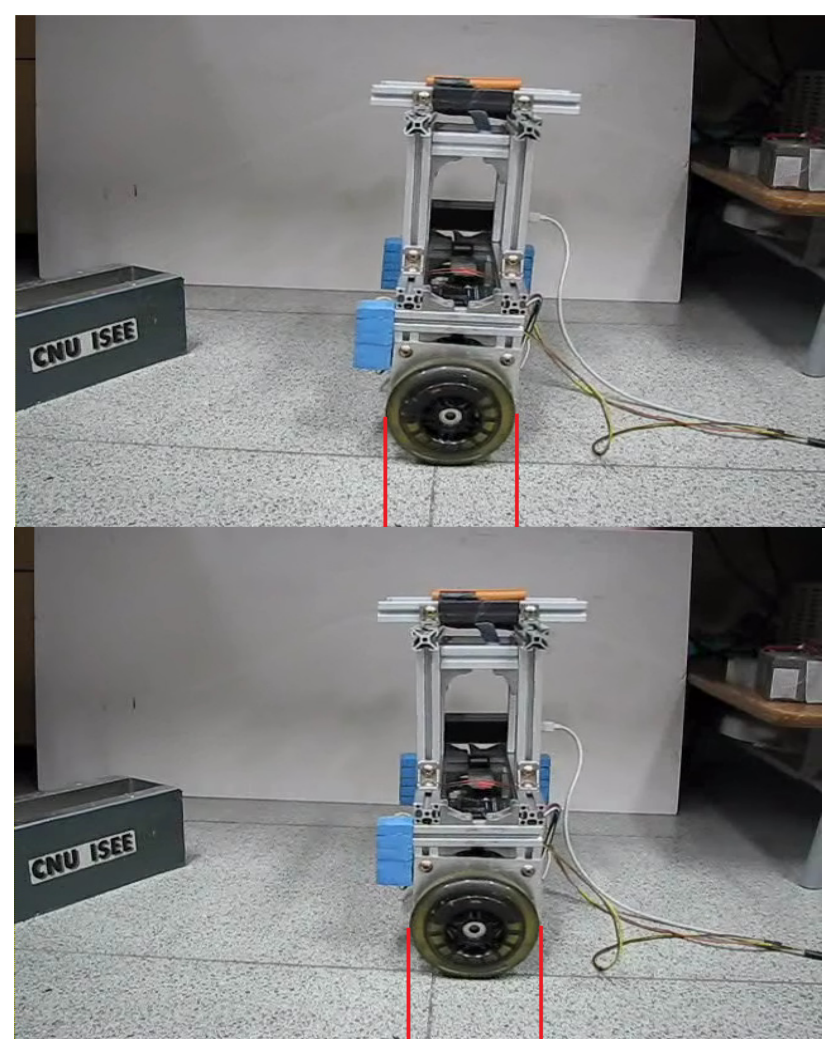

Fig. 8 Balancing demonstration by the linear controller 


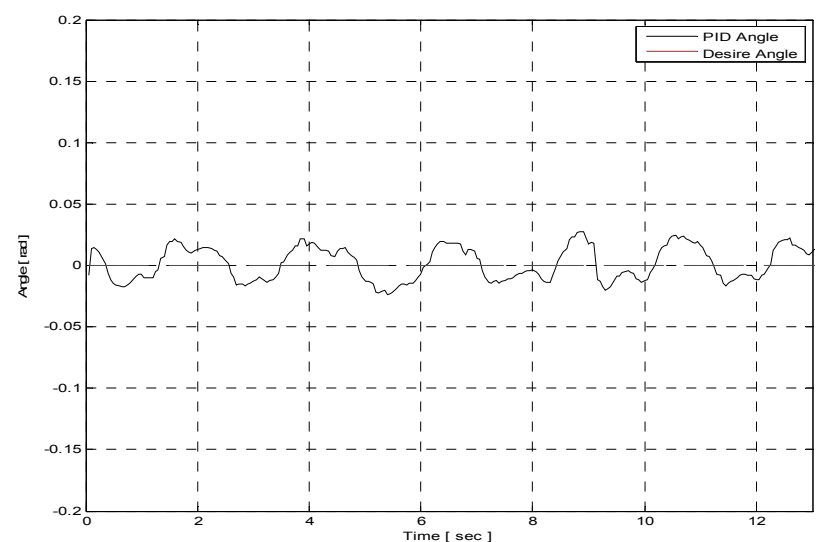

(a) Balancing angle

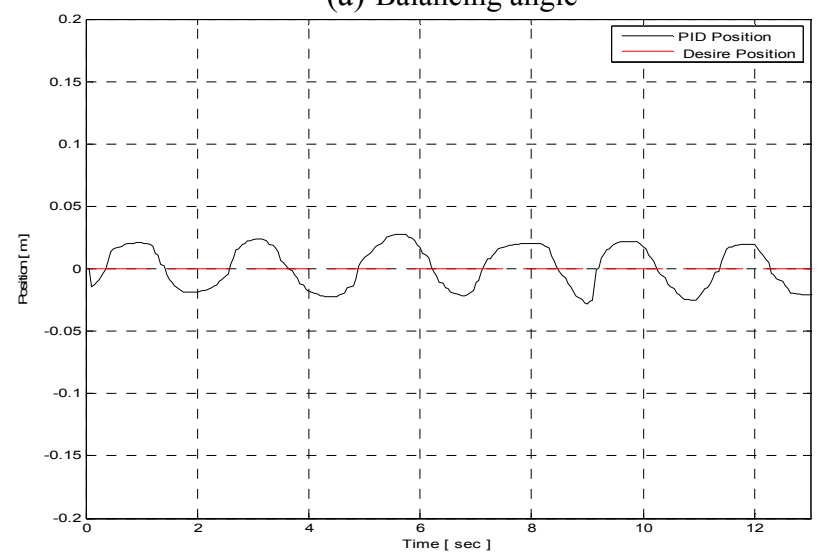

(b) Position

(c) Fig. 9 Balancing results by the linear controller

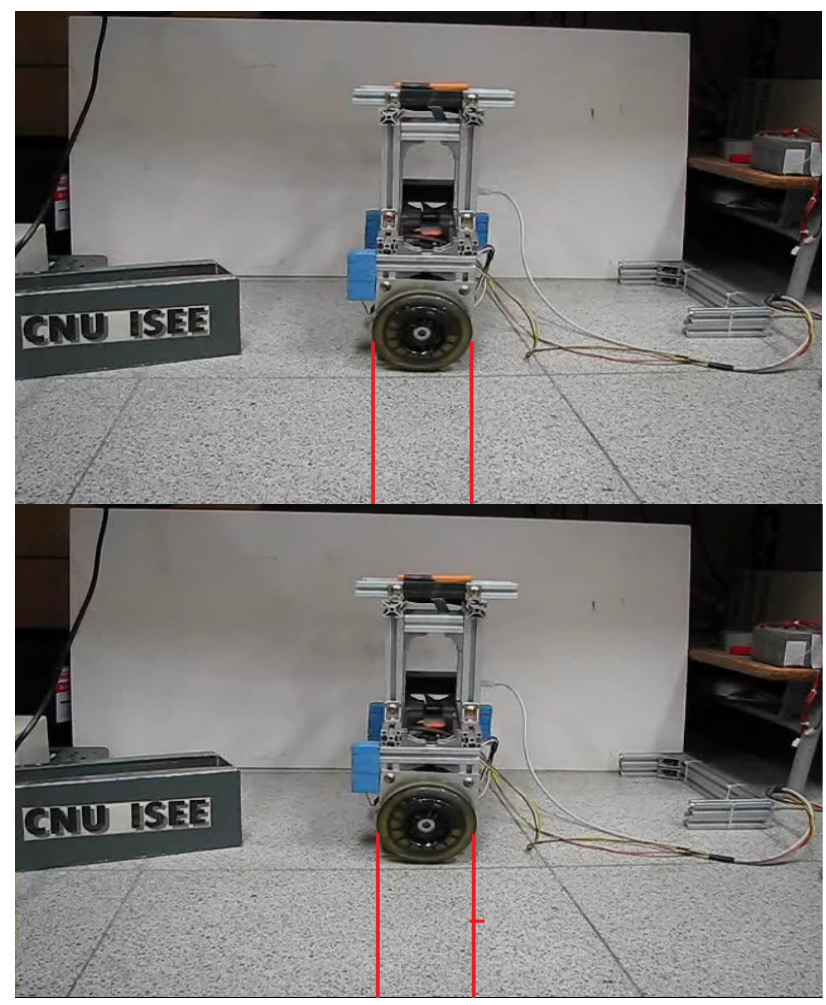

Fig. 10 Balancing demonstration by the Fuzzy controller
The fuzzy control method is also tested for the balancing performance as shown in Fig. 10. Red lines of two images are almost aligned, which means that TWMR does not oscillate much back and forth. The corresponding plots of Fig. 11 clearly explain that oscillatory movements are quite small that the positional error is less than $\pm 0.005 \mathrm{~m}$. A balancing angle error, a position error, and a heading angle error plot are shown in Fig. 11 (a), (b), and (c), respectively. The fuzzy controller works quite well such that the TWMR system is maintaining balance within small balancing angle error bounds of \pm 0.05 radian.

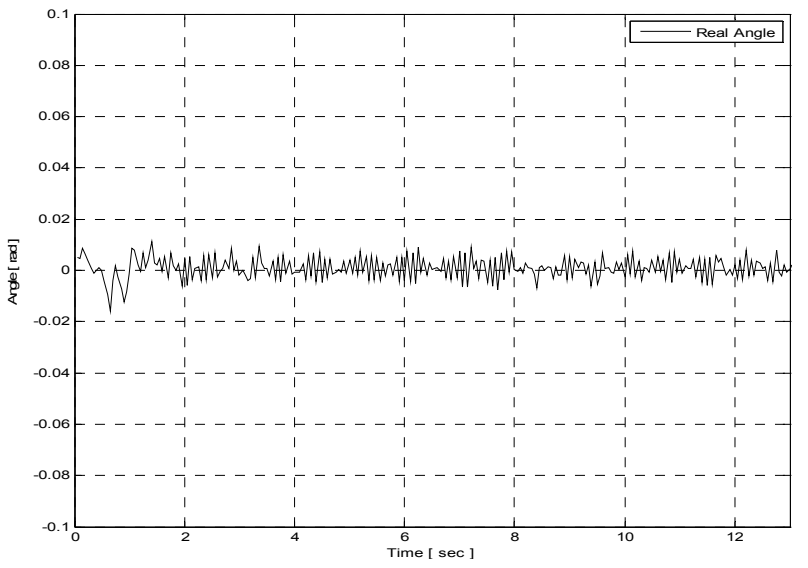

(a) Balancing angle error

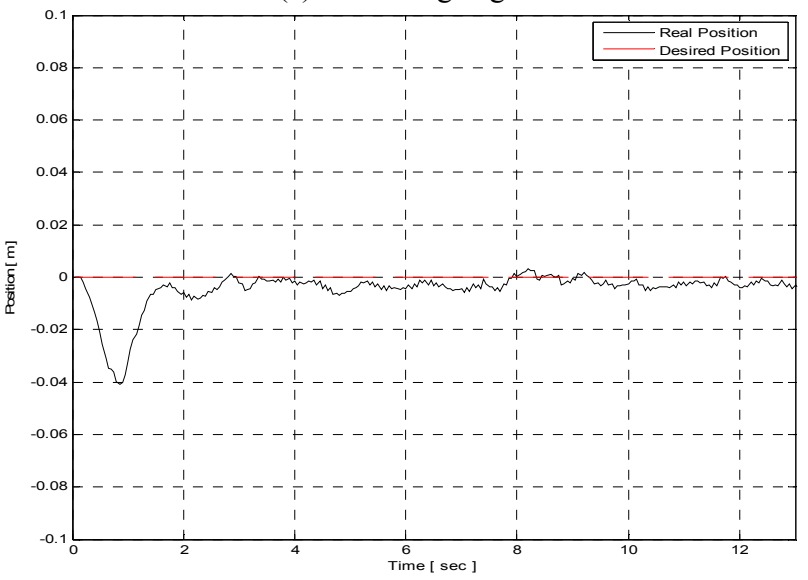

(b) Position error

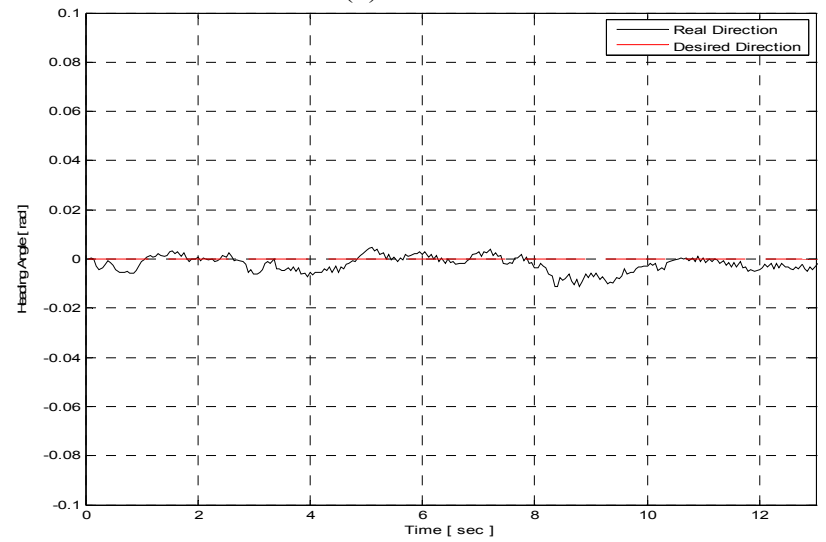

(c) Heading angle error

Fig. 11 Balacing results by the fuzzy controller 
To clearly demonstrate the perfromance between the linear control and fuzzy control, we plot them together as shown in Fig. 12. We clearly see the errors of the linear control method larger than those of the fuzzy control method.

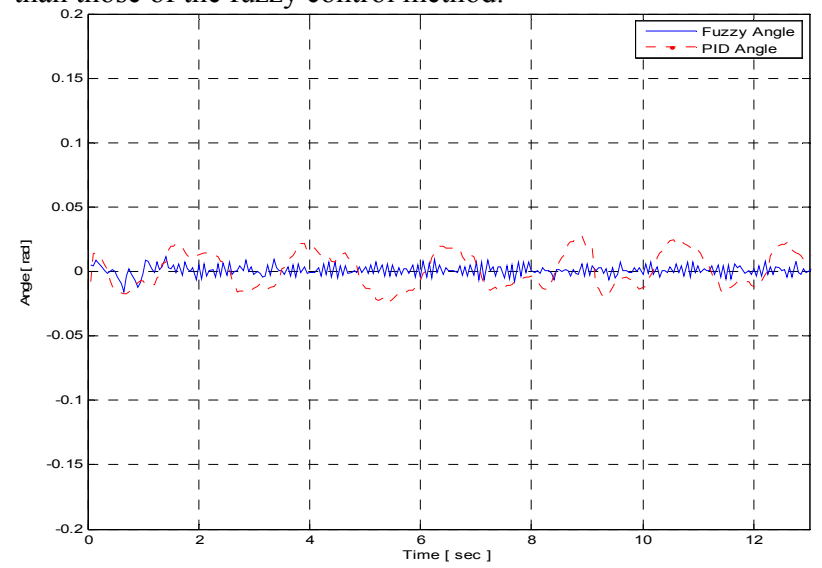

(a) Balancing angle

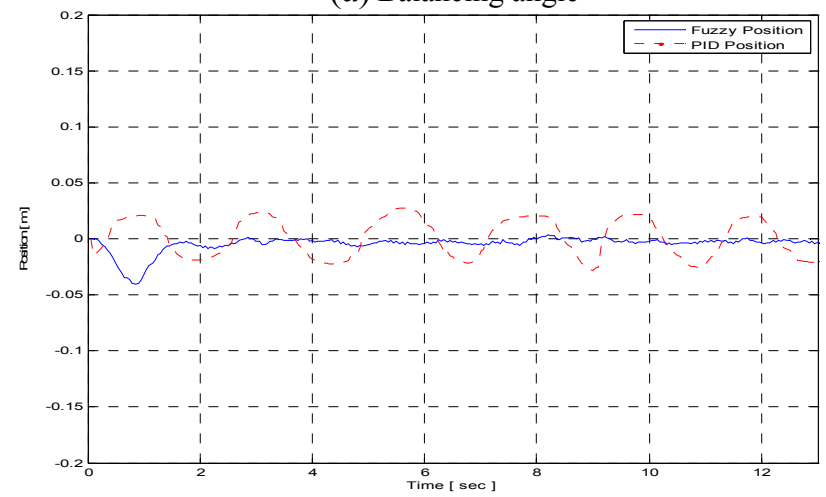

(b) Position

Fig. 12 Balacing performances by Two controllers

\subsection{Balancing Control Experiment under Disturbance}

Next experiment is to test the robustness of the fuzzy controller. The TWMR is intentionally hit by hands to check the balancing performances as in Fig. 13. Three external hits are applied to the system and the corresponding balancing responses are observed as shown in Fig. 14.

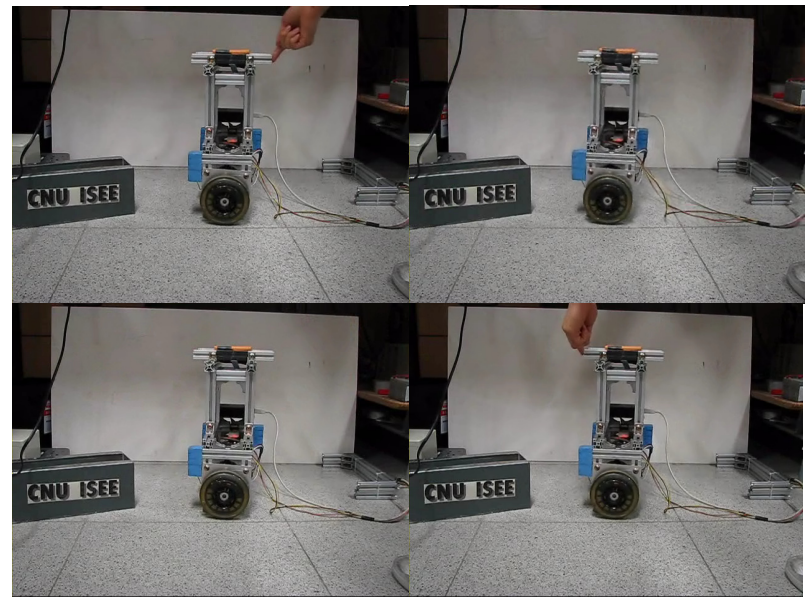

Fig. 13 Image cut under disturbance by fuzzy control
We clearly see from Fig. 14 that the TWMR immediately maintains balance after each intentional hit. Balancing performances by a fuzzy control method are robust enough to handle disturbances. Overshoots of angles in Fig. 14(a) are deviated angles from zero. Overshoots of the position in Fig. 14(b) demonstrate that the robot moves back and forth.

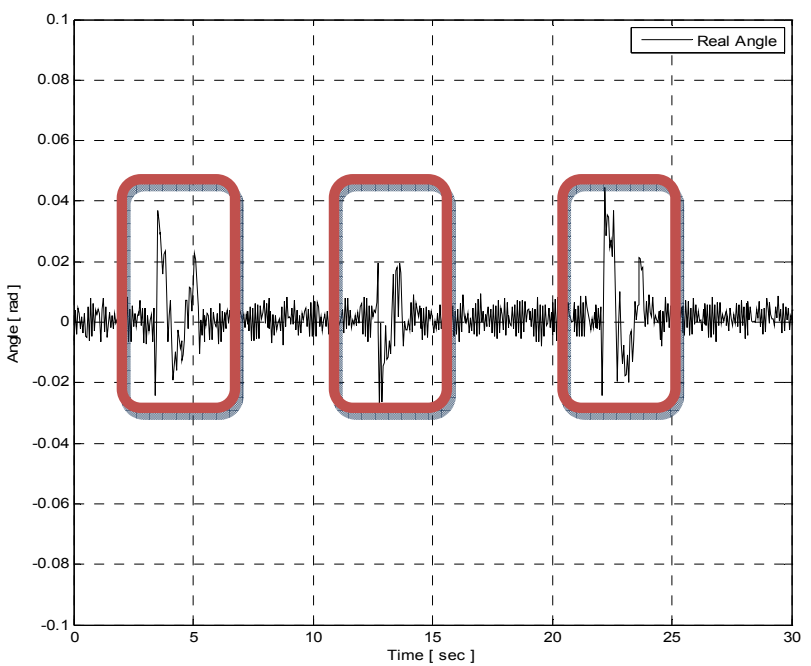

(a) Balancing angle error

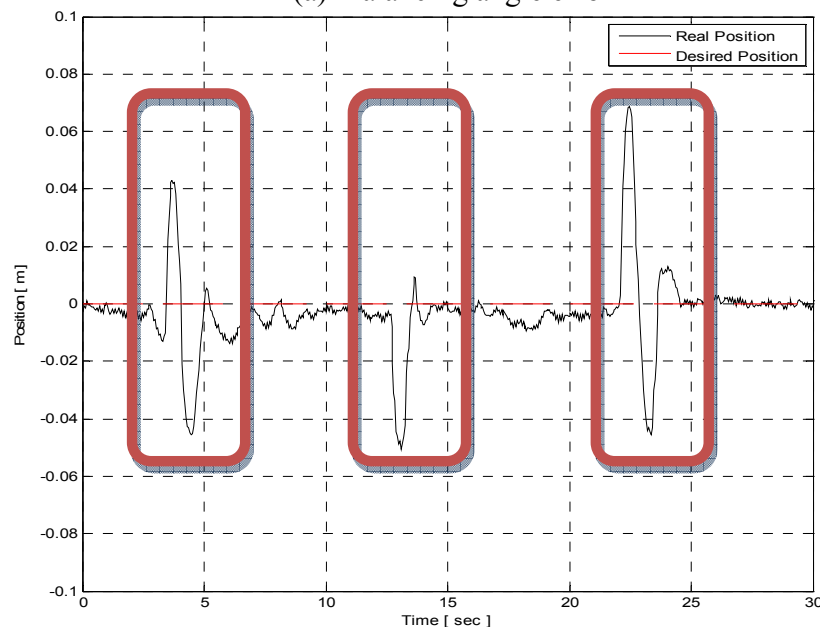

(b) Position error

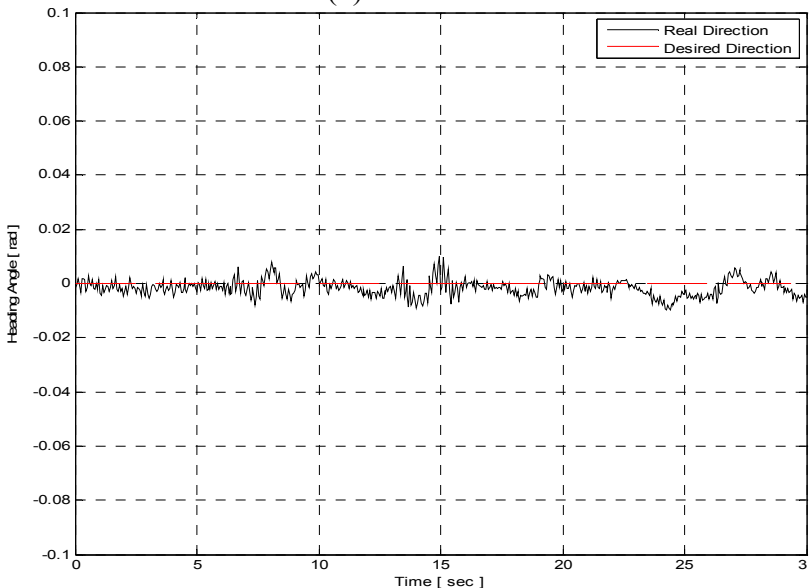

(c) Heading angle error

Fig. 14 Results under disturbance by fuzzy control 


\subsection{Comparison of Balancing Control Experiment} between PID control and fuzzy control

Experimental comparison studies between the linear control method and the fuzzy control method are presented. Fig. 15 shows the comparison plots of balancing performance under external disturbances. Although each hit to TWMR cannot be exactly same, it is reasonably applied to be equal.

The linear control method is able to balance the system under the disturbance, but we clearly see from Fig. 15 (a) that angle overshoots for the linear control method due to intentional hits are larger than those by the fuzzy control method.

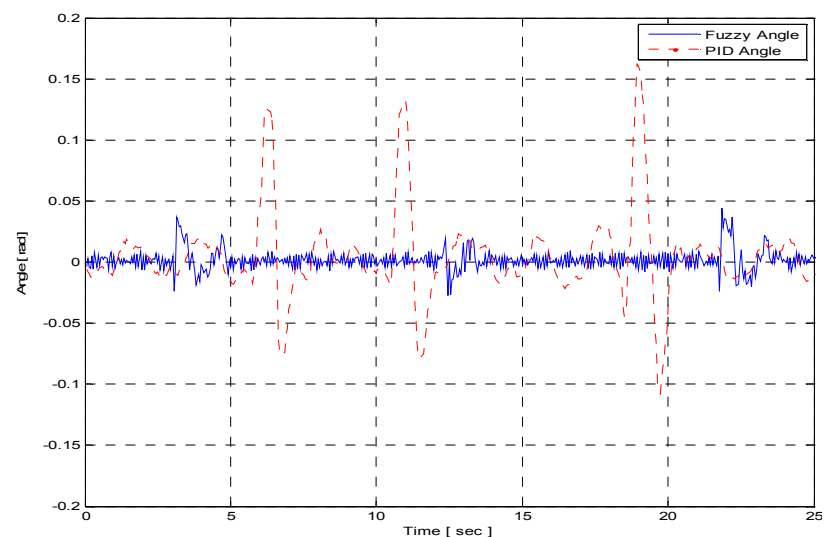

(a) Balancing angle error

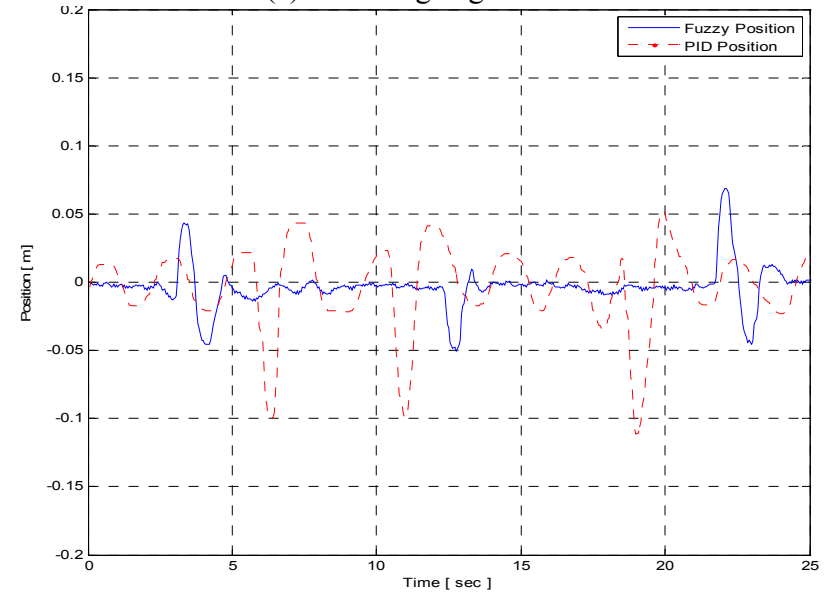

(b) Position error

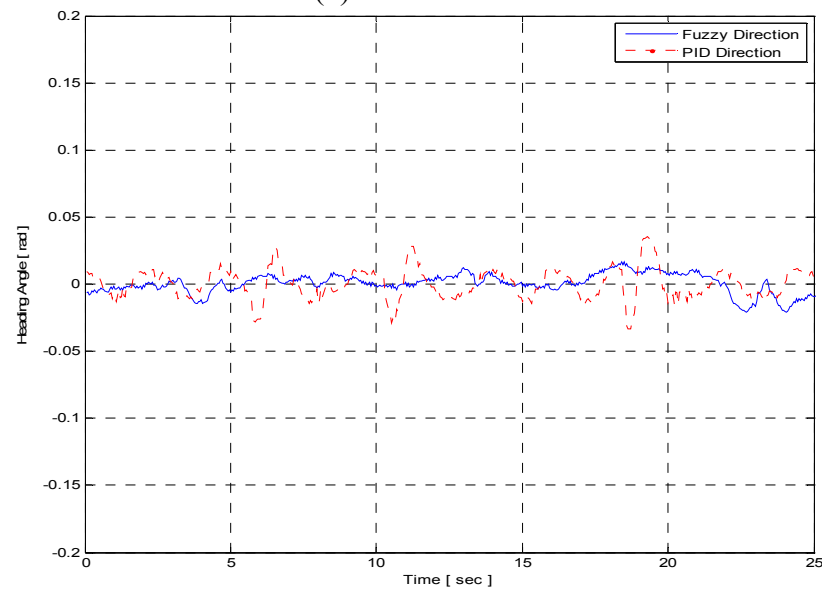

(c) Heading angle error
Fig. 15 Comparison of balancing control results between linear control method and fuzzy control method

\section{Conclusion}

This paper delivers the comparison studies of balancing performances of the two-wheel mobile robot system by the linear control and the fuzzy control method. TWMR system is implemented as a test-bed. Results of extensive experimental studies support the statement that the PID control method works well, but the fuzzy control method works better in the sense of robustness against external disturbance. Although nominal fuzzy rules instead of optimized rules are used for experimental studies, the fuzzy controller shows the better balancing performance especially when intentional hits are applied to the system.

In the future research, fuzzy rules can be optimized further to minimize the computational time.

\section{Acknowledgements}

This research has been partially supported by Korea Research Fund through the basic research program and the center for autonomous intelligent manipulation (AIM) for service robots of the MKE (The Ministry of Knowledge Economy), Korea, under the Human Resources Development Program for Convergence Robot Specialists support program supervised by the NIPA (National IT Industry Promotion Agency) (NIPA-2011-C7000-1001-0003).

\section{References}

[1] A. Sekiguchi, K. Kameta, Y. Tsumaki, and D. N. Nenchev, "Biped walk based on vertical pivot motion of linear inverted pendulum," IEEE Conf. on Advanced Intelligent Mechatronics, pp. 1-6, 2007

[2] Z. Tang and M. J. Er, "Humanoid 3D gait generation based on inverted pendulum model," IEEE Symposium on Intelligent Control, pp. 339-344, 2007

[3] "Segway," http://www.segway.com

[4] F. Grasser, A. Darrigo, S. Colombi, and A. Rufer, "JOE: A mobile, inverted pendulum," IEEE Trans. on Industrial Electronics, vol. 49, no. 1, pp. 107-114, 2002.

[5] K. Pathak, J. Franch, and S. K. Agrawal, "Velocity Control of a Wheeled Inverted Pendulum by Partial Feedback Linearization," IEEE Conference on Decision and Control, pp. 3962-3967, 2004

[6] S. M. Boskovich, "A two-wheel robot control system," IEEE WESCON 1995

[7] S. H. Jeong and Takayuki Takahashi, "Wheeled Inverted Pendulum Type Assistant Robot : Inverted Mobile, and Sitting Motion," IEEE/RSJ International Conference on Intelligent Robots and Systems, pp. 1932-1937, 2007. 
[8] K. Pathak, J. Franch, and S. Agrawal, "Velocity and position control of a wheeled inverted pendulum by partial feedback linearization," IEEE Trans. on Robotics, vol. 21, pp. 505-513, 2005.

[9] R. Imamura, T. Takei, and S. Yuta, "Sensor drift compensation and control of a wheeled inverted pendulum mobile robot," IEEE Workshop on Advanced Motion Control, pp. 137-142, 2008.

[10] J. Angeles, "An innovative drive for two wheeled mobile robot," IEEE/ASME Trans. On Mechatronics, vol. 10, no. 1, pp. 43-48, 2005.

[11] P. Oryschuk, A. Salerrno, A. M. Al-Husseini, and J. Angeles, "Experimental validation of an underactuated two-wheeled robot," IEEE/ASME Trans. on Mechatronics, vol. 14, no. 2, pp. 252-257, 2009.

[12] S. S. Kim and S. Jung, "Control experiment of a wheel-driven mobile inverted pendulum using neural network," IEEE Trans. on Control Systems Technology, vol. 16, no. 2, pp. 297-303, 2008.

[13] C. C. Tsai, H. C. Huang, and S. C. Lin, "Adaptive neural network control of self-balancing two-wheeled scooter," IEEE Trans. on Industrial Electronics, vol.57, no.4, pp. 1420-1428, 2010.

[14] C. H. Huang, W. J. Wang, and C. H. Chiu, "Design and implementation of fuzzy control on a two-wheel inverted pendulum system," IEEE Trans. on Industrial Electronics, vol.58, no.7, pp. 2988-3001, 2011.

[15] H. Tirmant, M. Baloh, L. Vermeiren, T. M. Guerra, and M. Parent, "B2, An alternative two wheeled vehicle for an automated urban transportation system," IEEE Intelligent Vehicle Symposium, pp. 594-603, 2002

[16] P. K. W. Abeygunawardhana and T. Murakami, "Vibration suppression of two-wheel mobile manipulator using resonance-ratio-control-based null-space control," IEEE Trans. on Industrial Electronics, vol.57, no.12, pp. 4137-4145, 2010.

[17] R. O. Ambrose, R. T. Savely, S. M Goza, P. Strawser, M. A. Diftler, I. Spain, and N. Radford, "Mobile manipulation using NASA's robonaut," IEEE Conf. on Robotics and Automations, pp. 2104-2109, 2004.

[18] K. Teeyapan, J. Wang, T. Kunz, and M. Stilman, "Robot Limbo: Optimized planning and control for dynamically stable robots under vertical obstacles," IEEE Conf. on Robotics and Automations, pp. 4519-4524, 2010.

[19] K. Sasaki and T. Murakami, "Pushing operation by two-wheel inverted mobile manipulator," IEEE Workshop on Advanced Motion Control, pp. 33-37, 2008.

[20] P. K. Abeygunawardhana and M. Toshiyuki, "Environmental interaction of two wheeled mobile manipulator by using reaction torque observer," IEEE Workshop on Advanced Motion Control, pp. 348-353, 2008.
[21] R. Imamura, T. Takei, and S. Yuta, "Sensor drift compensation and control of a wheeled inverted pendulum mobile robot," IEEE Workshop on Advanced Motion Control, pp. 137-142, 2008.

[22] J. Angeles, "An innovative drive for two wheeled mobile robot," IEEE/ASME Trans. On Mechatronics, vol. 10, no. 1, pp. 43-48, 2005.

[23] Y. S. Xu and K. W. Au, "Stabilization and path following of a single wheel robot," IEEE/ASME Trans. On Mechatronics, vol. 9, no. 2, pp. 407-419, 2004.

[24] H. J. Jin, J. M. Hwang, and J. M. Lee, “A balancing control strategy for a one wheel pendulum robot based on dynamic model decomposition : simulation and experiments," IEEE/ASME Trans. On Mechatronics, vol. 16, no. 4, pp. 763-768, 2011.

[25] P. Oryschuk, A. Salerrno, A. M. Al-Husseini, and J. Angeles, "Experimental validation of an underactuated two-wheeled robot," IEEE/ASME Trans. On Mechatronics, vol. 14, no. 2, pp. 252-257, 2009.

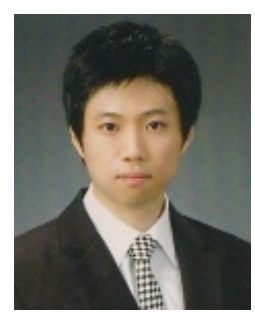

Hyun Wook Kim

He received his B.S. and M.S degrees in Mechatronics Engineering from Chungnam National University in 2008 and 2011, respectively. He is now at LIG Nex1. His research interests are intelligent control applications and control hardware design, DSP systems, and balancing robot systems.

Phone: $+82-42-821-7232$

Fax : +82-42-823-4919

Email:kwh1042@naver.com

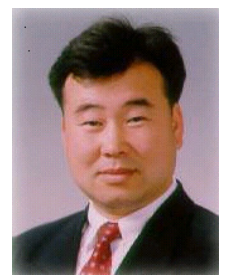

\section{Seul Jung}

He received his B.S. degree in Electrical \& Computer Engineering from Wayne State University in 1988, and his M.S. and Ph.D. degrees in Electrical \& Computer Engineering from the University of California, Davis in 1991 and 1996, respectively. He joined the Department of Mechatronics Engineering, Chungnam National University in 1997, where he is presently a professor. His research interests include intelligent control systems, robot education, and intelligent robotic systems.

Phone: $+82-42-821-6876$

Fax : +82-42-823-4919

Email: jungs@cnu.ac.kr 